\title{
Research on the Novel Mode of Plane Graphic Design from the Angle of Visual Language Transmitting
}

\author{
Hui Wu \\ Art Institute Of Jiujiang University, \\ Jiujiang ,Jiangxi,332000 China
}

\begin{abstract}
In this paper, we conduct research on the novel mode of plane graphic design from the core angle of the primary visual language transmitting. Relationship between nationality and cosmopolitan processing in graphic design in the design of image symbol expression and significance of the symbol on the one hand require symbolic form must have enough openness and the cognitive function on the other hand with semiotics method can let the designer to extract the most representative and symbolic notation style to carry on the design expression. As a non-verbal symbols, from the perspective of the view, the design image is no national boundaries, but the human form to beauty and should express the symbolism of cognition is the same. In the form of the ethnic groups of the cosmopolitan codes are for identification of Chinese contemporary design possible effective methods. Our research proposes novel perspective of the design which is meaningful.
\end{abstract}

Keywords- Novel Mode, Plane Graphic Design, Visual Language, Transmitting, General Angle.

\section{Introduction}

As part of the design art, graphic design is in a wide field of art. A good graphic design work, not only to the audience to good information from the United States, and more can make it taste Chinese brush over a long period of time, in the order of words and form color atmosphere, create some kind of their own imagination emotional sublimation that have profound. Graphics can play the important role it is inseparable features practicability and artistry. The first is practical. In the process of the development of human history, has formed a rich graphics system, a lot of graphics has a specific meaning, has the characteristics of vivid and symbolic, which makes graphics can beyond geographical, ethnic, various restrictions, such as the language of the world as the second is the artistic quality. Graphic elements with broad space performance with combination between different graphics can give person with the beauty of the novel visual feeling and rich experience, so as to strengthen the whole artistic quality.

Began to tend to attach the importance to people's emotional factors in the design, hoping to find a more accurate and clear semantics to strengthen the communication between users and products, as prompting the user for the product kind, trust and other positive emotional experience; At the same time want to use symbols show the symbolism of the deep connotation of graphic design, in order to make up for the lack of cultural connotation of modernistic design caused the situation. As the review of the literatures, we summarize the corresponding requirements as the follows. (1) The subconscious ideas. The formation of the subconscious ideas very fast natural it with designers' cultural quality of the high and low, reasonable ways of thinking and has its corresponding emotion space has a direct relationship, which requires the designer while learn many knowledge, and make the best use of their own life experience and the thing between transverse and primary longitudinal imagination space. (2) Creative inspiration. Creative inspiration is not produced by anytime and anywhere, it is designers in our daily life, careful observation of life and good at capturing the essential characteristics of things and fully exercises their imagination of thinking ability. The rich and colorful social life provides the 
digging endless source of inspiration for the designers. (3) Logical ideas. Logic the creativity is the designer used in graphic design is the most effective form of a kind of creative. It refers to the pattern design according to the logical thinking, as layers of in-depth development, in order to determine the performance of the theme of creative content and form [1-3].

Traditional handmade design material and as a visual sense of graphic design method as handmade completed due to material, so it makes his works on the simple primary sense of material has the very independent personality and natural sense of manual. Designers now many are themselves to look for making paper to do their own design. So it is possible to bring a lot of texture effect of the machine production does not exist. Life common visualization of the material texture using the visual sense is another kind of life, life material refining of visual element, build its texture effect of the consequent may be thick texture and the feeling of light visual language expression [4].

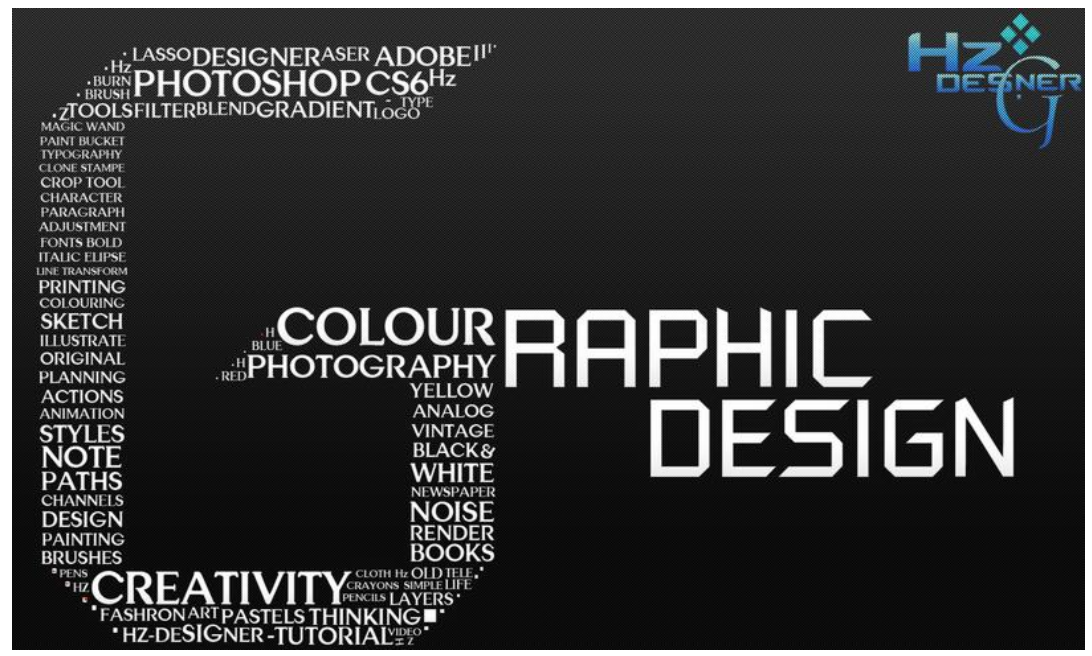

Figure 1. The Visualized Keywords of the Plane Graphic Design Principles

In this paper, we conduct research on the novel mode of plane graphic design from the angle of the visual language transmitting. Product itself is a form with will, expression and the communication of language function of comprehensive system is the embodiment of designers design ideas that is also a product of the embodiment of the practical function and aesthetic value. Product is not critical to the success of the technology, but in the very consistent products and the user's heart and emotions. In the later sub-sections, we will discuss the corresponding issues in detail.

\section{The Proposed Methodology and Perspective}

The Visual Language Transmitting. Visual communication of the important role is to make things more easily understood by people in the world, it is to use visual language to communicate with the people, visual communication as the information of complicated together with artistic image contains the artistic and philosophical expression for the perceived and get approval. It is a medium between the designer and the audience, through the organic combination of natural elements to the relatively strong visual impression. Visual communication design refers to through the general design of visual language to convey information, its wonderful delamination, firm soft and design features of powerful rich visual communication effect at the same time, also form and bring up the diversity strategy of the visual communication design, along with the basic role 
of the visual communication design in social development, the degree of this major teaching attention is self-evident [5-6].

The perception of the visual language is one of the visual languages parsing of thinking, to form the consciousness, get to experience. Understand the visual language perception process, can facilitate us to better the context of the new media of visual communication design of the visual language make interpretation that could be organized as the follows. (1) Personalized communication behavior. The personalized spread way making the personalized visual effects, selection of visual forms of the space increases the visual language that both audience bright individual character, and make its experience pleasure to release information to influence others. (2) Law and present form. New media in the spread of more abundant content, text, images, sound and other primary multimedia become a trend. Therefore, its visual presentation form and combinations are also diverse. (3) Interactive mode of the basic transmission. The spread of traditional media is one-way, linear, choose system. It concentrated expression is in a specific period of time by the information publishers dissemination of information to the audience passively accept, no of information feedback. The spread of this static model makes information liquidity and the spread of new media is a two-way, interactive, two-way here, on the one hand, refers to the traditional publishers and audiences are now become the publisher of information.

Visual communication design is not freely for the self-expression, but based on a specific case to a series of visual elements can be used for the transformation of the specific symbols, to achieve the effect of visual communication, so the visual communication design in teaching, we should pay much attention to visual communication design in the specific case analysis and interpretation of the visual communication design of the collect inside. Indeed, the visual communication designs in teaching we should consider the specific situation is three-dimensional as mutiple level, it is particularly important to the observer of the emotional experience that is reflected from the listed figure 2 .

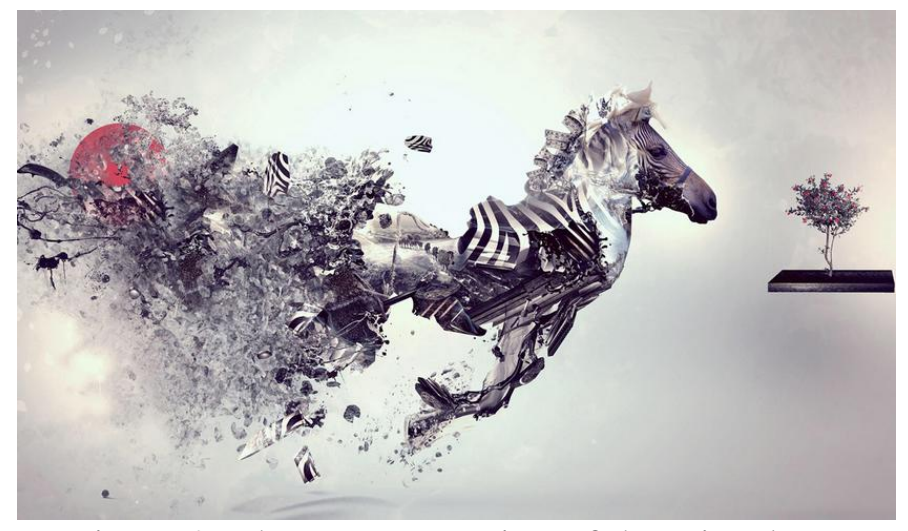

Figure 2. The Demonstration of the Visual Language Transmitting Concept

The Design Aesthetic. Graphic design success lies in the functional and aesthetic perfect reflect, this is inseparable from its form aesthetic feeling, and to form the aesthetic factors in addition to the human feelings, emotions should possess in the image thinking and rich emotion, it is also influenced by science and technology, ethic, economic and practical, and so on subjects.

The aesthetic feeling of design is still at the initial stage, in the discussion of aesthetics from a lot of theory combining with the design practice and design aesthetics has started a breakthrough. Graphic design in the design result is more and more big, the social function is more and more of the complex, increasingly exquisite beauty in the form of the graphic design is becoming more and more mature. Graphic design artistic charm of beauty is in the form of graphic design as the main factors that give a person with beautiful enjoyment, graphic design in the form of beauty are worth careful study.

Many traditional aesthetics is a sign of things to the nature color description, because of its special visual effect to the viewer bring different sensory experience, which is endowed with diverse human spirit emotion and symbolism, 
institutionalized become symbolic symbols and the schemata. Both the ancient art and modern graphic design, the symbolic meanings are interlinked. Such as mentioned above, different color is a symbol of the status of people, quality, character, etc., in the plane design, if the designer can according to work target people's information needs, cultural background, traits of the character, life taste and appreciate the level of information and its status can be chosen effectively, the quality and character of color as the main body color graphic works as it can greatly improve work attractive and information dissemination [7].

The Principles of the Visual Language. Visual thinking is a kind of visual image as the medium of operation and which has the function of rational thinking, and the visual image as the intermediary of thinking in essence is a kind of creative thinking. In researchers' view, creative visual thinking embodied in three aspects first of all, because of the visual thinking is the result of direct perception, which itself has exploratory from unknown to the known, subject can directly feel the fresh thinking source, and play a role of main body for free choice and the creation. Secondly, as a result of visual thinking is the basic operation unit or thinking tool of visual imagery, knowing subject can directly feel the fresh visual images, and the visual image can be free of regeneration and combination.

Graphic design graphics, with the image design illustration is the picture of the language, the visual language of creation, expression and arrangement, produce strong visual communication effect that promote the appeal of works, completing the process of visual communication can be organized as the follows. (1) Graphical illustrations. Graphic design of graphic illustration class gives person with the feeling of contemporary and concise simplified generalization abstraction plane processing technique to integrate multifarious things concise and, strengthen the main characteristics of theme. (2) Painting class illustrations as painting elements in graphic design with a casual, free and easy feeling. Painting elements varied, such as using different tools such as ink, charcoal with the stick performance design illustrations have different visual effect. Different painting language is suitable for different graphic design theme, for example, sports theme that is suitable for the painting with strong dynamic factor expression with implicit culture theme available ink painting expression effect. (3) Photography class illustrations. In graphic design photography illustration have intuitive visual perception. Photographic illustrations persuasive, close to reality as objectively reflect the life, some of the commonweal poster language through photography form intuitive performance theme, with a shock of visual effect.

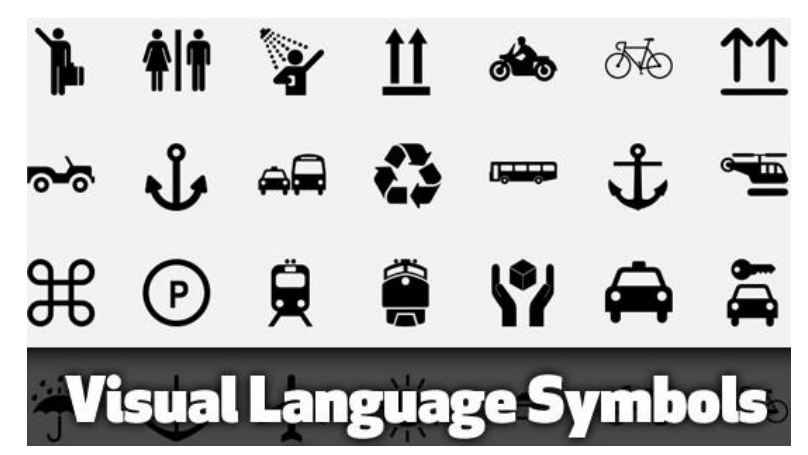

Figure 3. The Sample Demonstration of the Visual Language

The Future of Plane Graphic Design. In life often indicate type of the expression for example we see the tender green shoots will think of spring may come, feel the north wind roared, will feel the winter, not far from the psychological suggestion, in fact, this phenomenon is expressed by concept of a whole system center sign, this is human in the process of the long-term development through core summarizing the natural phenomena and life experience and formed a kind of symbol. And applied to the plane design is the auxiliary symbols in graphic design, graphic design work with the background information of convey are closely linked, with works of graphic symbol structure played an important role. Creativity is not only a kind of the 
design skills and more important is the nature of a design externalization. Creative besides talent, mainly at ordinary times the accumulation of experience and knowledge. From the depth, is the professional skills and the embodiment of the ideological content from the breadth, it is the accumulation of life experience, is itself to live, to society, to the feeling of life. The more abundant, the thought collision sparks, the more creative the opportunity. Design must be sincere, if the designer's design has no credibility that cannot meet the target consumers' cognitive habits, such ideas will not be able to stimulate, formation and improve the target consumers [8].

As image expression of design thinking, or abstract means, is inseparable from the application of symbols. In image thinking, designers through a general, vivid, indicative of the symbolic language to form to the real appearance of the system design, function and so on various aspects of meaning, and in the design thinking, abstract means it is with the aid of all kinds of abstract or not imitative symbols to form the concept of object and the significance of expression. The second design thinking is a kind of creative thinking. Often through the logo design of figure and bottom, the processing of positive and negative space, the dimensional feeling of the logo graphic performance through to the negative space artistic conception, transfer the negative space in enhancing people's visual interest, artistic conception and the extensibility and develop the positive role of the multiple design space, etc. The reasonable use of negative space and its artistic conception ably extension, effectively improve the modelling is the problem of insufficient space in logo design, alleviate the adverse factors of basic modelling, revealing signs of visual effect, and with the volume of space feeling promote graphic visual impact, help to modern information communicated adequately, make people feel the space of the plane figure and the fundamental beauty of it with reflections.

\section{Conclusion}

In this paper, we conduct research on the novel mode of plane graphic design from the angle of the visual language transmitting. Creativity is the most important in the plane design, is the key to design work. On the creative is the hardest. Creative, creative ambiguity, can create design is not successful, from the original intention. The grasp of the creative, in fact, a kind of view of the world culture for designers, only by constantly learning about the knowledge of the industry, to improve the quality of the individual culture can more easily grasp the creative thinking, to create a satisfactory work. The creativity is the soul of the work and it is a kind of creative thinking, to express their own creative perfect, and accepted by the audience is the key to creation. Creative work idea is unlimited, but show on different individuals, have different ways to understand in different design needs of creative will have different ways of expression. In the further research, we will combine more related issues and the knowledge to optimize the current perspective.

\section{References}

[1] Zhang, Shao Jie. "The Use of Computer Image Processing Technology in the Area of Graphic Design." Applied Mechanics and Materials. Vol. 687. Trans Tech Publications, 2014.

[2] Wook, Tengku Siti Meriam Tengku, and Siti Salwah Salim. "Guideline for the Graphic Design of Web Application for Children's Interface." TELKOMNIKA Indonesian Journal of Electrical Engineering 11.6 (2013): 3130-3133.

[3] Ping, W. A. N. G. "A Research on the Training of Motion Graphic Design Capability for Visual Communication Design Major." Journal of Zhejiang Wanli University 6 (2014): 018. 
[4] CHEN, Hang-ping, and Xue-wei FENG. "The Dynamic Expression of Static Plane Graphic on Screen [J]." Packaging Engineering 12 (2013): 008.

[5] Appiah, Edward, and Johannes Cronj. "Information Communication and Technology (ICT) and the Challenges of Ideation in Graphic Design: An Activity Theory Focus." International Journal of Computer Applications 63.6 (2013).

[6] Yunpeng, Zhao. "Application of" Problem Solving" Mode in the Teaching of Computer
Graphic Design." Office Informatization 2 (2013): 022.

[7] ZHAO, Jiahua, and Kexin CHEN. "Research of Project Teaching Based on Studios in Photoshop Graphic Design Course." Journal of Changsha University 2 (2013): 047.

[8] Gomes, Toni, et al. "The effect of full body versus partial body graphic labelling on beverage packaging." Packaging Technology and Science 27.12 (2014): 933-943. 